\title{
Modulating Specific Central and Peripheral Pathways to Effectively Treat Obesity
}

Chooi Yeng Lee*

School of Pharmacy, Monash University Malaysia

Keywords: Appetite centres; Gut hormones; High fat-diet; Stress; TLR4

Dysregulation of the homeostatic control of appetite and hepatic glucose production, and the imbalance of the interrelation between the gut microbiota and the innate immune system, are currently believed to be the main causes of obesity. Most of these mechanisms function by interacting with one another, rendering high levels of complexity in continual maintainance of a stable body weight. Understand the molecule that acts specifically or having a regulatory role in each of the pathway, is therefore crucial in effective management of obesity development. A number of function-specific mechanisms and pathways of the hypothalamus and the gut have been identified as potential therapeutic targets.

The arcuate nucleus (ARC) of the hypothalamus is particularly important in the integration of central and peripheral signals that regulate appetite. The ARC houses two neuronal circuits, ie. the appetite- and the satiety-stimulating circuits, which signal using specific neurotransmitters to modulate feeding behavior and energy expenditure. Gut hormones have long been recognised as the primary peripheral signals that determine the release of specific central neuropeptides [1]. Most recently, favourable outcome from bariatric surgeries has led to a rejuvenated interest in the gut and the gut-brain neuronal axis. Bariatric surgeries performed on obese, non-diabetic patients caused a reduction in acyl-ghrelin, and significant increase in anorexigenic peptides such as peptide $\mathrm{YY}_{3-36}\left(\mathrm{PYY}_{3-36}\right)$ and glucagonlike peptide (GLP)-1 [2]. Because bariatric surgery is currently the only clinically used obesity treatment that has resulted in significant long-term weight loss, these hormones may be critical in the regulation of body weight. However, ghrelin receptor is widely expressed in the central nervous system, and exerts different effects in different areas of the brain. Infusion of ghrelin receptor antagonist into the cerebral ventricles decreases caloric intake and weight gain [3], but chronic antagonism of ghrelin receptor in the paraventricular nucleus (PVN) increases the animal's preference for high fat-diet (HFD) and their body weight gain [4]. Therefore, unless the inhibitor of ghrelin receptor can be designed to reach brain regions other than the PVN, its use may be of limited beneficial effects. GLP-1 is effective in reducing appetite and body weight [5], but has unwanted side effect, ie. it may cause hypoglycaemia in non-diabetic subjects [6]. Compare with ghrelin and GLP-1, PYY ${ }_{3-36}$ may be a more promising target. Daily, intermittent intravenous infusion of PYY ${ }_{3-36}$ has been reported to cause a sustained reduction in daily food intake and body weight [7]. Since PYY has higher affinity to the ARC $\mathrm{Y}_{2}$ than other $\mathrm{Y}$ receptors, peripheral administration of suitable dose of $\mathrm{PYY}_{3-36}$ to specifically target $\mathrm{Y}_{2}$ receptor may be effective in long-term body weight control.

Besides appetite, several centrally mediated pathways have been reported to control glucose homeostasis [8]. One of the more established pathway involves long-chain fatty acyl-coenzyme A (LCFACoA). LCFA-CoA, a product of intestinal lipid metabolism, binds to $\mathrm{N}$-methyl-D-aspartate (NMDA) receptor in the fourth ventricle or the nucleus of the solitary tract and reduces hepatic glucose production [9]. In the cell, LCFA-CoA enters mitochondria via carnitine palmitoyl transferase (CPT)-1 and undergoes $\beta$-oxidation. Interestingly, pharmacological inhibition of the ARC's CPT1 decreases food intake and glucose production [10], while accumulation of LCFA-CoA in the hypothalamus lowers glucose production [11]. Data collectively suggest that glucose homeostasis can be effectively controlled via modulation of the NMDA receptor in the brainstem, and inhibition of CPT1 in the ARC.

There is a developing interest on gut microbiota, where extensive research in this area has offered a different yet wider perspective on the cause of obesity and treatment option. Lipopolysaccharides (LPS) is a major component of the Gram-negative bacterial outer membrane. It binds to toll-like receptor (TLR)-4, a receptor expressed in the intestinal epithelial cells and adipose tissues [12]. Intestinal TLR4 is vulnerable to overactivation because any change in the gut microbiota composition causes an increase in luminal LPS [13]. TLR4 activates nuclear factor-kappa B and increases the expression of pro-inflammatory cytokines. Mucosal inflammation alters the tight junctions, which enhances intestinal permeability, subsequently systemic and tissues inflammation [13]. Cytokines expressed in the adipose tissues and liver are associated with the onset of insulin resistance, and HFD-fed TLR4 knockout mice were protected from these pathological conditions [12]. Moreover, administration of LPS induces obesity and visceral adiposity to the same extent as HFD feeding [14]. In short, obesity is associated with inflammation, and TLR4 inhibition can suppress the development of many hallmark features of obesity.

HFD and psychosocial stress are two major risk factors of obesity. HFD alters the composition of gut microbiota [11] and attenuates intestinal lipid sensing mechanisms [8], including the mechanism initiated by LCFA-CoA [9]. Psychosocial stress promotes the release of glucocorticoids and ghrelin. In fact, plasma ghrelin increases in parallel with glucocorticoids [15] and the duration of stress [16]. Chronically stressed mice had an increased adiposity and weight gain, consistent with elevated levels of ghrelin, and hypothalamic neuropeptide Y and agouti-related peptide expression [3]. Rodent introduced to physical and psychological stress also suffered from intestinal mucosal inflammation and degeneration, intestinal barrier dysfunction, and impaired mucosal defence against infectious agents $[17,18]$. Recently, I have shown that chronic stress alters the expression profile of intestinal nutrient transporters [18]. Particularly interesting was the up-regulation of facilitative glucose transporter-2 (SLC2A2, formerly

*Corresponding author: Chooi Yeng Lee, School of Pharmacy, Monash University Malaysia, Jalan Lagoon Selatan, 47500 Bandar Sunway, Selangor, Malaysia, Tel: +60355146314; Fax: +60355146323; E-mail: chooi.yeng.lee@monash.edu

Received November 23, 2013; Accepted December 26, 2013; Published January 02,2014

Citation: Lee CY (2014) Modulating Specific Central and Peripheral Pathways to Effectively Treat Obesity. J Mol Genet Med S1: 007. doi: 10.4172/1747-0862.S1-007

Copyright: (C) 2014 Lee CY. This is an open-access article distributed under the terms of the Creative Commons Attribution License, which permits unrestricted use, distribution, and reproduction in any medium, provided the original author and source are credited 


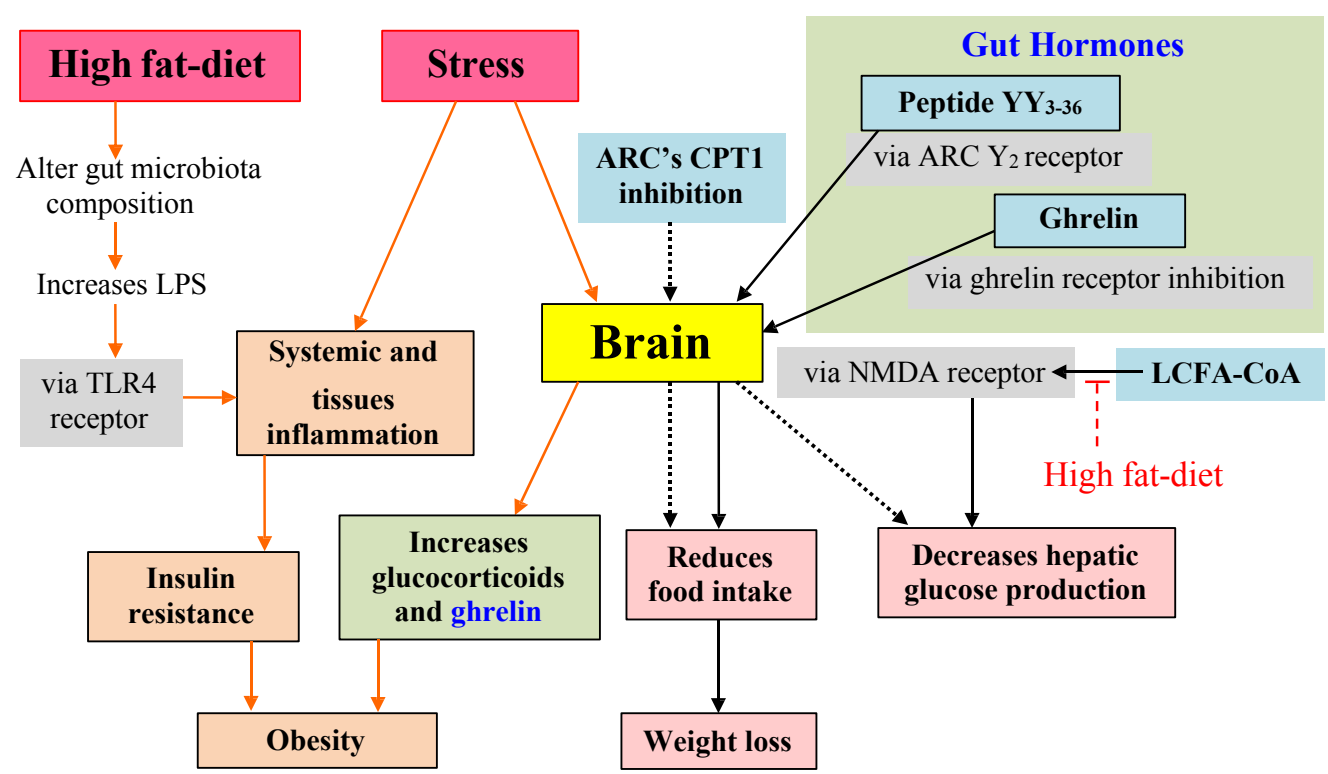

Figure 1: Targeting specific mechanisms and pathways to counteract the effects of high fat-diet and stress. Peptide YY3-36 induces satiety by binding preferentially to the arcuate nucleus (ARC) $Y_{2}$ receptor. Inhibition of the hypothalamic ghrelin receptor (except that in the paraventricular nucleus) and the ARC's carnitine palmitoyl transferease (CPT)-1 reduces food intake and glucose production (dotted lines refer to the effects from CPT1 inhibition). Intestinal long-chain fatty acyl-coenzme A (LCFA-CoA) binds to the brainstem's N-methyl-D-aspartate (NMDA) receptor and decreases hepatic glucose production. This lipid sensing mechanism is attenuated by high fat-diet (HFD) (red dashed line). HFD and stress via activation of the toll-like receptor (TLR)-4 and the release of ghrelin, respectively, causes inflammation and obesity (orange lines represent the impact of HFD and stress). Modulation of these molecules (boxes in blue) and receptors (boxes in grey) are potential strategies to control body weight gain, (LPS) lipopolysaccharides.

known as GLUT2) in the duodenum, which seemed to be correlated with inflammation and morphological changes of the enterocytes. There may be many other physiological function of the gut that contributes to obesity development but is yet to be revealed. Because HFD exaggerates response to stress [19], stress-induced pathophysiological changes will be enhanced in the presence of HFD. Figure 1 shows some of the major mechanisms and pathways that regulate energy balance and body weight, and how HFD and stress disrupt the homeostasis.

In summary, modulation of ghrelin and $\mathrm{PYY}_{3-36}$ levels, the expression of central CPT1 and NMDA receptor, and peripheral TLR4 receptor are specific therapeutic strategies that may increase the effectiveness of obesity treatment. Most importantly, these mechanisms and pathways are bound to be disrupted by HFD and stress. Managing these function-specific molecules may be useful to tackle the major risk factors of obesity.

\section{References}

1. Murphy KG, Bloom SR (2006) Gut hormones and the regulation of energy homeostasis. Nature 444: 854-859.

2. Yousseif A, Emmanuel J, Karra E, Millet Q, Elkalaawy M, et al. (2014) Differential effects of laparoscopic sleeve gastrectomy and laparoscopic gastric bypass on appetite, circulating acyl-ghrelin, peptide YY3-36 and active GLP-1 levels in non-diabetic humans. Obes Surg 24: 241-252.

3. Patterson ZR, Khazall R, Mackay H, Anisman H, Abizaid A (2013) Central ghrelin signaling mediates the metabolic response of $\mathrm{C} 57 \mathrm{BL} / 6$ male mice to chronic social defeat stress. Endocrinology 154: 1080-1091.

4. Patterson ZR, Parno T, Isaacs AM, Abizaid A (2013) Interruption of ghrelin signaling in the PVN increases high-fat diet intake and body weight in stressed and non-stressed C57BL6J male mice. Front Neurosci 7: 167.

5. Zander M, Madsbad S, Madsen JL, Holst JJ (2002) Effect of 6-week course of glucagon-like peptide 1 on glycaemic control, insulin sensitivity, and beta-cell function in type 2 diabetes: a parallel-group study. Lancet 359: 824-830.
6. Todd JF, Stanley SA, Roufosse CA, Bishop AE, Khoo B, et al. (2003) A tumour that secretes glucagon-like peptide-1 and somatostatin in a patient with reactive hypoglycaemia and diabetes. Lancet 361: 228-230.

7. Chelikani PK, Haver AC, Reeve JR Jr, Keire DA, Reidelberger RD (2006) Daily, intermittent intravenous infusion of peptide $Y Y(3-36)$ reduces daily food intake and adiposity in rats. Am J Physiol Regul Integr Comp Physiol 290: R298-305.

8. Lee CY (2013) The effect of high fat-diet-induced pathophysiological changes in the gut on obesity: what should be the ideal treatment? Clin Transl Gastroenterol 4: e39.

9. Wang PY, Caspi L, Lam CK, Chari M, Li X, et al. (2008) Upper intestinal lipids trigger a gut-brain-liver axis to regulate glucose production. Nature 452: 10121016.

10. Obici S, Feng Z, Arduini A, Conti R, Rossetti L (2003) Inhibition of hypothalamic carnitine palmitoyltransferase-1 decreases food intake and glucose production. Nat Med 9: 756-761.

11. Caspi L, Wang PY, Lam TK (2007) A balance of lipid-sensing mechanisms in the brain and liver. Cell Metab 6: 99-104.

12. Shi H, Kokoeva MV, Inouye K, Tzameli I, Yin H, et al. (2006) TLR4 links innate immunity and fatty acid-induced insulin resistance. J Clin Invest 116: 30153025.

13. de La Serre CB, Ellis CL, Lee J, Hartman AL, Rutledge JC, et al. (2010) Propensity to high-fat diet-induced obesity in rats is associated with changes in the gut microbiota and gut inflammation. Am J Physiol Gastrointest Liver Physiol 299: G440-448.

14. Cani PD, Amar J, Iglesias MA, Poggi M, Knauf C, et al. (2007) Metabolic endotoxemia initiates obesity and insulin resistance. Diabetes 56: 1761-1772.

15. Asakawa A, Inui A, Kaga T, Yuzuriha H, Nagata T, et al. (2001) A role of ghrelin in neuroendocrine and behavioral responses to stress in mice. Neuroendocrinology 74: 143-147.

16. Lutter M, Sakata I, Osborne-Lawrence S, Rovinsky SA, Anderson JG, et al. (2008) The orexigenic hormone ghrelin defends against depressive symptoms of chronic stress. Nat Neurosci 11: 752-753.

17. Ercan F, Cetinel S, Contuk G, Cikler E, Sener G (2004) Role of melatonin in 
Citation: Lee CY (2014) Modulating Specific Central and Peripheral Pathways to Effectively Treat Obesity. J Mol Genet Med S1: 007. doi: 10.4172/17470862.S1-007

Page 3 of 3

reducing water avoidance stress-induced degeneration of the gastrointestinal mucosa. J Pineal Res 37: 113-121.

18. Lee CY (2013) Chronic restraint stress induces intestinal inflammation and alters the expression of hexose and lipid transporters. Clin Exp Pharmacol
Physiol 40: 385-391.

19. Pascoe WS, Smythe GA, Storlien LH (1991) Enhanced responses to stress induced by fat-feeding in rats: relationship between hypothalamic noradrenaline and blood glucose. Brain Res 550: 192-196. 